DOI: $10.1007 / \mathrm{s} 00350-009-2479-6$

\section{Medizinische Ethik. Gesundheitsschutz, Selbstbestimmungsrechte, heutige Wertkonflikte.}

Von Hartmut Kreß. Verlag W. Kohlhammer, 2. Aufl. Stuttgart 2009, $326 \mathrm{~S}$., kart., $€ 34,00$

Kaum mehr als fünf Jahre nach dem Erscheinen seiner „Medizinischen Ethik" (2003) hat der Bonner evangelische Theologe und Sozialethiker Hartmut Kreß deren 2. Auflage (2009) vorgelegt. Doch für die sich stürmisch entwickelnde Biomedizin, die im Zentrum dieses Arbeits- und Studienbuches steht, ist ein halbes Jahrzehnt eine lange Zeit. Viel gab es in Teil A zu den normativen Leitlinien, mehr noch im aktualitätsbezogenen Teil B mit seinen gesetzgebungs-relevanten Gebieten einzuarbeiten. Der Umfang wuchs dabei von 208 auf 326 Seiten, für deren Erschließung ein Sachregister von mehr als 2 Seiten zu wünschen wäre. Das Spektrum dieser Medizinethik mit ihren kompetent dargestellten medizinischen Fakten reicht von den theologischen, philosophischen und kulturgeschichtlichen Grundlagen über gesellschaftliche, politische und rechtliche Wertungen bis hin zu der kritisch kommentierten Gesetzeslage und Reformdiskussion in der Biomedizin. Durch Mitarbeit in wichtigen Gremien, wie der Berliner Stammzell-Kommission, der Bioethik-Kommission Rheinland-Pfalz oder dem Wissenschaftlichen Beirat der Bundesärztekammer (BÄK), gewährleistet der Autor den rechtlich-politischen Praxisbezug seiner Medizinethik.

Im Vordergrund der Entwicklung seit der 1. Auflage steht die Novellierung des Stammzellgesetzes (StZG) mit ihrer Verschiebung des Stichtags vom 1.1.2002 auf den 1.5.2007. Kreß präzisiert seine damaligen Bedenken: ein starrer Stichtag sei sukzessiv forschungshemmend und stehe sachlogisch in Widerspruch zur Dynamik der Forschung und Entwicklung. Zugleich kritisiert er, dass das Gesetz zwar begrenzt Forschung erlaube, aber die Anwendung von Forschungsergebnissen verbiete, etwa zur pharmakologischen oder toxikologischen Nutzung. Der Autor beklagt zu Recht moralische Doppelstandards, da das StZG die Erzeugung von Stammzellen aus Embryonen im Inland ausschließe, aber den Import aus dem Ausland begrenzt zulasse. Umstrittene neue Ansätze in der Stammzellforschung, die auf den Verbrauch früher Embryonen verzichten wollen, beurteilt er ethisch vorsichtig positiv - z. B. therapeutisches Klonen durch Zellkerntransfer, Parthenogenese oder Depotententialisierung embryonaler Gebilde.

Auch die nach dem Embryonenschutzgesetz (ESchG) von 1990 erlaubte Fortpflanzungsmedizin beruht, wie Kreß betont, auf Embryonenforschung, und zwar solcher im Ausland, während sie im Inland verboten sei. Er plädiert für eine Zulassung der verbreitet als verboten geltenden Präimplantationsdiagnostik (PID), die als kleineres Übel einer späten Abtreibung vorzuziehen sei. Eindringlich stellt e die Mehrlingsschwangerschaften als Folge der künstlichen Befruchtung dar. Deren Risiken für Frau und Kind könnten weitgehend vermieden werden: durch den im Ausland längst praktizierten SingleEmbryo-Transfer (SET) nach morphologischer Auswahl möglichst nur eines Embryos. Wie schon die Gynäkologengesellschaft 2005 und die BÄK 2006, appelliert der Autor zu Recht an den bezüglich des ESchG schon lange säumigen Gesetzgeber, für dieses umstrittene Verfahren endlich Rechtsklarheit und Rechtssicherheit zu schaffen. Allerdings dürfte seine Aussage, die morphologische Beobachtung sei mit dem ESchG unvereinbar, nicht genügend den Auslegungsspielraum nutzen, den der enge Embryobegriff des Gesetzes eröffnet; denn dessen Merkmal „,entwicklungsfähig“ begrenzt den gesetzlichen Schutz und somit die Strafbarkeit.

Dieses gesetzliche Merkmal stellt Kreß in den philosophischen Kontext der Potentialität, die als Begründung für einen starken Embryonenschutz gilt. Zu Recht hält er dieses Argument für nicht tragfähig, zumal „Entwicklungsfähigkeit“ heute manipulierbar sei; der Gesetzgeber solle das Merkmal aufgeben. Statt dessen billigt der Autor dem Frühembryo nur einen minderen Sonderstatus mit graduell ansteigender Schutzwürdigkeit zu. Dieses gradualistische Prinzip, gegen das die 1. Auflage noch Bedenken formulierte, eröffnet Spiel-

Min.-Rat a.D. Dr. iur. Rudolf Neidert,

Wachtberg, Deutschland raum für Güterabwägungen in Reproduktionsmedizin und Stammzellforschung; umgekehrt folgt für den heranwachsenden Fötus eine wachsende Schutzverpflichtung des Staates. Konsequent fordert Kre $\beta$, der das Querschnittsthema Kindeswohl vor und nach der Geburt in der 2. Auflage noch stärker betont, für die Spätabtreibung lebensfähiger Kinder eine neue Befristung. Der Bundestag hat sich inzwischen jedoch zu kaum mehr als verstärkter Beratung bereit gefunden.

Neben diesen Wertkonflikten am Lebensbeginn können hier die vom Autor behandelten Problembereiche des Lebensendes nur erwähnt werden. Außer der Transplantationsmedizin ist dies vor allem die Sterbehilfe, die der Autor in einem stark erweiterten Kapitel behandelt. Kurz vor Ende der Wahlperiode kam es im Bundestag doch noch zu einem Gesetz, im freiheitlichen Sinne von Kreß. Wie in der 1. Auflage (Rezension Neidert in JZ 14/2004) werden diese Spezialbereiche von den kulturellen und normativen Grundlagen her interpretiert. Normativer Kern der Medizinethik ist für den Autor das Recht auf Gesundheitsschutz, das er nachdrücklicher noch als 2003 mit dem Grundrecht auf Freiheit und Selbstbestimmung verbindet, vielfältig belegt aus der Philosophie- und Theologiegeschichte. So trägt diese Medizinische Ethik die Prägung einer liberalen Wertargumentation, die gerade dem Medizinjuristen bei der so oft umstrittenen Auslegung biomedizinrechtlicher Vorschriften als Kompass dienen kann. Auch der Gesetzgeber sollte bei den von Kreß mehrfach angemahnten Reformen auf diese Fundgrube rechtspolitischer Leitbegriffe und kulturgeschichtlicher Werte zurückgreifen.

\section{Medizinrecht in der Onkologie (Klinische Onko- logie 2009/2010).}

Herausgegeben von Dirk Olzen, Helmut Frister und Stephan Roth. University Press, Düsseldorf 2009, 80 S., kart., $€ 10,00$

Die Heinrich-Heine-Universität Düsseldorf hat im März 2009 ein interdisziplinäres Symposium zur klinischen Onkologie in Düsseldorf durchgeführt. Der vorliegende Tagungsband enthält die 15 medizinischen und juristischen Beiträge. Diese betreffen die rechtlichen und betriebswirtschaftlichen Rahmenbedingungen eines medizinischen Versorgungszentrums (Möller und Michels). Thiele befasst sich mit der Wirksamkeit und Wirtschaftlichkeit neuer onkologischer Untersuchungs- und Behandlungsmethoden. Besonders wichtig für Onkologen erscheinen die Beiträge zum Off-Label-Use (Ufer) und zum Leistungsumfang der GKV bei der Behandlung von Patienten in klinischen Studien (Heyll), ferner die Beiträge zur Sterbehilfe (Grenzsituationen der Intensivmedizin) (Lindemann), Umfrageergebnisse zur passiven Sterbehilfe in einer Klinik (Möller/Grabensee/Frister). Patientenverfügung und Vorsorgevollmacht (Olzen), Beweisprobleme im Arzthaftungsprozess (Giesler) und Behandlungsfehler in der Gynäkologischen Onkologie (Weber/Beck) sowie Patientenaufklärung (Schwarz) runden die medizinischen Themen mit juristischen Auswirkungen ab. Kratz befasst sich mit der klinischen Prüfung von Arzneimitteln, Deiters erläutert den Codex der freiwilligen Selbstkontrolle für die Arzneimittelindustrie, Ohmanns Beitrag befasst sich mit nichtkommerziellen klinischen Studien.

Nicht alle Beiträge lassen sich hier kommentieren. Eines ist jedoch festzustellen: Trotz des breiten Spektrums ist es den Herausgebern gelungen, praktische Erfahrungen und wissenschaftliche Erkenntnisse zur klinischen Onkologie durch kundig ausgewählte Referenten hoch komprimiert zu vermitteln. Alle Beiträge sind gleichartig aufgebaut: Zunächst eine Zusammenfassung mit den Kernaussagen, dann der jeweils gut gegliederte Text, abgerundet durch weiterführende Literaturhinweise am Schluß des Beitrages.

Der Sammelband ist ein wertvolles Kompendium, das für die klinische Praxis wichtige Hinweise geben kann. Auch niedergelassene Onkologen sowie Anwälte, die sich mit der Beratung von Onkologen befassen, sollten es nutzen. 